\title{
The Toxicology Tower of Babel: Why We Need to Agree on a Lexicon in Prescription Opioid Research
}

\author{
Lewis S. Nelson • Leonard J. Paulozzi
}

Published online: 21 September 2012

(C) American College of Medical Toxicology 2012

\section{A foolish consistency is the hobgoblin of little minds, adored by little statesmen and philosophers and divines. $R W$ Emerson}

Ralph Waldo Emerson notwithstanding, inconsistency in the use of medical terminology, particularly in written communication, can lead to confusion. Propagation of even subtle variations in terminology and definitions through the literature can lead to inaccurate descriptions of medical problems and poor medical decision making that impact patient outcomes. In the medical literature, terms are often used interchangeably or are redefined by study authors to fit the context of their research methodology. Although there are bona fide needs to redefine terms in various study settings, accuracy and precision in the use of language is critical. This is particularly evident in the opioid analgesic literature because of the diverse backgrounds of those who write on the topic, including epidemiologists and clinicians, and their diverse clinical perspectives, ranging from pain medicine to addiction.

As we were preparing our own publications and reviewing those of our colleagues for this issue, the need for a consistent set of terms to describe the gamut of prescription opioid-related issues came sharply into focus. Even if authors define the terms, it is likely that many casual readers

\section{S. Nelson ( $ه)$}

New York University School of Medicine,

455 First Avenue, Room 123,

New York, NY 10016, USA

e-mail: lewis.nelson@nyumc.org

\section{J. Paulozzi}

Division of Unintentional Injury Prevention,

National Center for Injury Prevention and Control,

Centers for Disease Control and Prevention,

601 Sunland Park Drive, Suite 200,

El Paso, TX 79912, USA

e-mail: 1bp4@cdc.gov will apply their own preconceived definitions to many commonly used words.

We decided to explore some of the more challenging complications of the language used in the opioid analgesic literature and in regulatory use. This list is intended to be representative of the complexity of opioid analgesic language in the field, rather than a comprehensive treatment of the issue. We also offer potential solutions to this vexing and persistent problem.

\section{Why Are We Having Problems with Terminology?}

This entire issue of the Journal of Medical Toxicology is devoted to adverse events related to prescription opioids. Therefore, at the most basic level, we need to agree on what an "opioid" is. An opioid is a chemical that binds to, and stimulates (is an agonist at), opioid receptors in the brain and other tissue. The category includes endogenous opioids (e.g., endorphin, for endogenous morphine), opiates (e.g., opium-derived natural compounds such as morphine and codeine), semisynthetic opioids (compounds derived from opiates such as hydrocodone, oxycodone, and heroin), and fully synthetic opioids (e.g., methadone and fentanyl).

Although a word such as "opioid" had a medical definition, the medical and legal definitions of words are frequently confused. For example, although a "narcotic" in medical terminology represents a drug that has sleep-inducing properties, the term is far more widely interpreted in its legal meaning, where it includes drugs with very different properties, such as cocaine. The term "narcotic analgesic" is also widely and usually inappropriately applied to opioid analgesics. It implies "illegal" use and is best avoided in the medical literature. This recommendation should also extend to patient interactions because the majority of patients associate the word "narcotic" with its legal meaning. We need to separate the legal context of such analgesics (i.e., as 
narcotics) from their medical categorization as opioids based on their receptor affinities.

A number of terms describe how drugs are used, and distinctions among such terms have also become more important. As we have moved into an era of prescription drug abuse from an era of abuse of street drugs, attention to semantic subtleties is now perhaps even more important. For heroin, use, misuse, and abuse did not need to be distinguished. For opioid analgesics, we need to distinguish all three terms plus terms such as "nonmedical use." Similarly, the same chemicals can change labels depending on the circumstances. For example, a chemical can be described as a substance, a poison, a drug, or a medication depending on its original purpose, the intent of its current use, and the outcome of use.

The term "illicit drug use" is also ambiguous because either the drug or the use might be illicit, a distinction that was never necessary for heroin. Even when what is illicit is the type of use, illicit use of opioid analgesics can be hard to define. An opioid analgesic can certainly be obtained by prescription licitly but then used for illicit purposes. But what if it is obtained illegally but used for approved indications? Or even more complicated, what if it is obtained legally and used as indicated but obtained from five different doctors? As a result, when a publication uses the phrase "illicit drug use," it is unclear whether the behavior includes only drugs such as heroin, cocaine, and marijuana, or those drugs in addition to legal prescription drugs used in illicit ways.

Our attempts to be clear about the use of prescription drugs have sometimes created awkward constructions. For example, we frequently read the term "prescription opioid," but some object even to this simple label because much of the current epidemic involves prescription drugs that are not prescribed to their ultimate users. As a result, some have proposed the term "prescription-type" opioids, recognizing both that such drugs are used without prescription and that the alternative "non-prescription opioid" has a different set of problems. In this case, we have added even more terminology in a search for greater accuracy or precision.

To further complicate an already muddled terminology, we have problems with how we use and define the indication for opioid analgesics. For example, the phrase "pain medication" may seem paradoxical because it does not cause pain; it relieves pain. And the distinction between cancer pain and noncancer pain is far less clear than the phrases suggest.

One barrier to addressing the varying preferences for terminology is the poor quality of information available on the drug source and reason for use, especially in "complex" patients. Rarely is a person with a drugrelated problem willing to answer 20 or more questions about their circumstances of drug use. Therefore, even with the clearest and most distinct definitions available, a researcher cannot easily determine which classification to use to describe the problem. He or she might then opt for broad labels such as "misuse/abuse" and modifiers such as "possible" and "probable" and show a general lack of enthusiasm for elaborating even more specific terms.

\section{What Can Be Done?}

We advocate for a consistent labeling system for the medications themselves and a consistent taxonomy for mode of use for prescription drugs. We need to strive for descriptors that can handle the complexities of prescription drug use while remaining consistent with the definitions of terms already in use in medical settings. Terms should also be developed for situations that are currently poorly defined.

To provide a credible lexicon that encompasses multiple stakeholder perspectives, we suggest that a multidisciplinary working group be convened to properly frame the terminology and its use. This group should include (the lists are incomplete and in alphabetical order) nurses, pharmacists, and physicians, specializing in addiction, emergency medicine, forensic medicine, internal medicine, medical toxicology, pain medicine, and pediatrics. Epidemiologists and other researchers should be intimately involved, both from the private sector and from government agencies focused on public health, such as the CDC, FDA, NIDA, and SAMHSA.

In the same way that languages have developed dialects over time, opioid analgesic terminology has evolved in different disciplines to the point that we no longer understand one another clearly as we try to construct a solution to a shared, growing problem with prescription drugs. We need to first learn to speak a common language. We might have to tear down the current Tower of Babel and start over, but otherwise we won't be able to work together to address the problem effectively.

Conflict of Interest The authors have no conflicts of interest. 